\title{
Rapid Sensory Profiling of Tennis Rackets ${ }^{\dagger}$
}

\author{
Maximilian Bauer 1,*, Sean Mitchell ${ }^{1}$, Nathan Elliott ${ }^{2}$ and Jonathan Roberts ${ }^{1}$ \\ 1 Wolfson School of Mechanical, Electrical and Manufacturing Engineering, Loughborough University, \\ Loughborough LE11 3QF, UK; s.r.mitchell@lboro.ac.uk (S.M.); j.r.roberts@lboro.ac.uk (J.R.) \\ 2 R\&D Racquet Sports, HEAD Sport GmbH, 6921 Kennelbach, Austria; n.elliott@head.com \\ * Correspondence: m.p.bauer@lboro.ac.uk; Tel.: +44-7902-592-659 \\ + Presented at the 13th Conference of the International Sports Engineering Association, Online, \\ 22-26 June 2020.
}

Published: 15 June 2020

\begin{abstract}
Tennis racket manufacturers rely on subjective assessments from testers during the development process. However, these assessments often lack validity and include multiple sources of inconsistency in the way testers make subjective ratings. The purpose of this research was to investigate the suitability of the free-choice profiling (FCP) method in combination with principle component analysis (PCA) and multiple factor analysis (MFA) to determine the sensory profile of rackets. FCP was found to be a suitable technique to quickly evaluate the sensory profile of rackets; however, consumer testers tended to use ill-defined, industry-generated terms, which negatively impacted discrimination and inter-rater agreement. Discrimination and inter-rater agreement improved for attributes referring to measurable parameters of the rackets, such as vibration. This study furthers our understanding of tennis racket feel and supports racket engineers in designing new subjective testing methods, which provide more meaningful data regarding racket feel.
\end{abstract}

Keywords: feel; sensory analysis; tennis rackets; free-choice profiling; PCA; MFA

\section{Introduction}

Since performance advancements have become more challenging in the tennis racket industry, manufacturers rely increasingly on consumer feedback in terms of racket feel to provide direction in the development process. The feel of a piece of sports equipment has also been highlighted as a major factor influencing a consumer's buying decision [1]. As a result, manufacturers incorporate user perception into the development process of new rackets and rely on the meaningfulness of subjective data. Due to the complex nature of the game, however, subjective assessments of racket feel often lack reliability and include multiple sources of inconsistency.

Previous research on feel in tennis has explored certain parameters in isolation, such as moment of inertia [2] and sound [3]. These studies focused on establishing links between subjective and objective data. However, no study to date has investigated the reliability and suitability of methods used to gather subjective data.

Sensory evaluation of consumer products has been traditionally separated into analytical tests, performed by trained panels, and hedonic tests using consumers [4]. Racket brands constantly update their products, therefore internal development decisions must be made quickly and the time-consuming process of training a panel is not desirable. In the food industry, sensory evaluation techniques are heavily used and the use of consumers for analytical evaluation of sensory characteristics has lately gained appreciation [4].

As a result, so-called rapid sensory profiling techniques have evolved [5], with one of the most popular methods being free-choice profiling (FCP) [6]. FCP allows testers to self-define a series of attributes on which they wish to judge the sample of products, based on their initial perceptions of 
the most salient features. This addresses the issue of testers being forced to rate rackets according to attributes defined and deemed suitable by the investigator. FCP also offers the advantage of assessing the sensory characteristics of a sample of products in a single session, whilst providing the most prominent, marketable terms at the same time.

The main aim of this study, therefore, is to evaluate the suitability of FCP in determining the sensory profile of tennis rackets. FCP will be considered suitable if

1. Common attributes emerge amongst multiple testers (attribute prominence).

2. Testers are able to differentiate consistently between rackets with regard to a particular attribute (discrimination capability).

3. Multiple testers judge the rackets in the same way on the most prominent attributes (degree of agreement).

Application of statistical techniques, such as principal component analysis (PCA) or multiple factor analysis (MFA) in the context of subjective data analysis from tennis racket tests, will also be discussed. Increasing knowledge about novel sensory evaluation methods in tennis research will help racket engineers to make more efficient decisions in the development process by applying methods to obtain more meaningful on-court data. Accurate determination of sensory profiles of rackets will allow feel characteristics to be tailored to the consumer and could even be used to enhance marketing strategies.

\section{Materials and Methods}

Thirty-eight male tennis players were recruited to perform the FCP task (age $=45 \pm 10$ years). The group of participants consisted of recreational tennis players, playing at least once a week but not competitively. This specific demographic group of tennis players was used because they best represent the target group of the rackets under investigation. All tests were performed on the outdoor clay courts of two local clubs in Vorarlberg, Austria.

\subsection{Rackets}

Four different rackets (A, B, C, and D) were evaluated. The exact specifications of the rackets are confidential; however, the ranges within which they fall are given in Table 1. All rackets were less than $295 \mathrm{~g}$ in mass and differed considerably in head sizes, stiffness, balance, swing weight, and frame geometry. All rackets were strung at $24 \mathrm{~kg}$ with the same string, equipped with string dampers and had a $16 \times 19$ string pattern. Rackets exhibiting a broad spectrum of varying characteristics were chosen over rackets only differing in one characteristic; as such, a selection would evoke a wider range of racket-specific vocabulary, making it particularly suitable for FCP.

Table 1. Racket specifications described by the minimum and maximum value of all rackets.

\begin{tabular}{ccc}
\hline Racket Parameter & Minimum & Maximum \\
\hline Head Size $\left[\mathrm{cm}^{2}\right]$ & 645 & 670 \\
Mass $[\mathrm{g}]$ & 282.3 & 292.3 \\
Balance Point $[\mathrm{mm}]$ & 305.5 & 326.8 \\
Swing Weight $\left[{\left.\mathrm{kg} \times \mathrm{cm}^{2}\right]}^{2}\right.$ & 273 & 279 \\
Stiffness ${ }^{1}[\mathrm{~Hz}]$ & 117 & 151 \\
\hline tiffness represented by the frequency of the first bending mode.
\end{tabular}

\subsection{Free-Choice Profiling Protocol}

The unique characteristic of FCP is that testers can define their own attributes. After a five minutes warm-up, all testers were given instructions on how to perform the task. Four testers were tested at a time on two adjacent courts. A duplicate set of rackets was produced, in order to allow smooth flow of the protocol. On each court, two testers shared one of the two identical sets of rackets. 
The testers were all German speaking, and all attributes mentioned in this study were translated from German into English by the lead investigator, who is fluent in both languages.

Testers were allowed to provide between one and four attributes. They were encouraged to only select attributes, for which they perceived the largest differences between rackets. Each tester had to play with each racket at least once before making their first rating and they could swap rackets as many times as they wanted to. If testers experienced difficulties in verbalizing perceived differences, the investigators supported them in finding appropriate descriptors.

The ratings were recorded using $100 \mathrm{~mm}$ Visual Analogue Scales (VAS) with ends marked by a minus and a plus. To aid understanding, testers could add word anchors to either end of the scale and provide descriptions of the self-defined attributes.

\subsection{Statistical Analysis}

Terms and descriptions for the different attributes used by the testers were compared, and those that were considered to share a similar theme were grouped together. The percentage of testers that used each sensory attribute was selected as a measure of attribute prominence. Only the most prominent attributes, which were mentioned by at least six (>15\%) testers, were analyzed further.

The discrimination capability of testers on the most prominent attributes was determined by the $p$-values from Friedman's two-way analysis of variance (ANOVA) by ranks and by the mean ranges of the particular VAS scale being used. To assess the degree of agreement between each tester and the group consensus, profile plots were used. Fleiss' kappa was used as an objective measure of inter-rater agreement.

Finally, PCA was used to determine the relationship between sensory attributes. PCA was performed on a $4 \times 10$ matrix with one score per racket per attribute. Racket specifications and the overall liking of the rackets, assessed at the end of each session, were added as additional variables. Since averaging causes inter-tester variance to be lost and because of the occurrence of different attributes, MFA is a more common approach in FCP data analysis. Therefore, data were also analyzed by MFA and all scores were standardized by subtracting the mean and dividing it by the standard deviation. Both MFA and PCA were performed with the Rstudio software (version 1.1.463, RStudio, Inc., Boston, MA, USA) using the FactoMineR package [7].

\section{Results}

The attributes most frequently used to describe the rackets are summarized in Table 2. The table also highlights how different but related terms were grouped together and given a shared attribute name. The $p$-values from Friedman's test are shown in Table 3. The smaller the $p$-value, the more likely it is that rackets were perceived as being different. This test has only been performed on attributes exceeding the minimum recommended number of testers.

Table 2. Frequency of sensory attributes and (translated) terms used by the testers to rate the rackets.

\begin{tabular}{llc}
\hline \multicolumn{1}{c}{ Sensory Attribute } & \multicolumn{1}{c}{ Initial Terms Used with Translations } & Percentage Used \\
\hline 1. Power & Power, Pace, Speed, Zug (pull), Beschleunigung (acceleration) & $74 \%$ \\
2. Control & Präzision (precision), Kontrolle (control) & $61 \%$ \\
3. Vibration & Vibration & $40 \%$ \\
4. Sound, 5. Heaviness & Klang (sound); Kopflastigkeit (heaviness), Gewicht (weight); & $21 \%$ each \\
6. Stiffness & Steifigkeit (stiffness) & $18 \%$ \\
7. Forgiveness, 8. Spin, & Fehlerverzeihend (forgiveness); (Top-) Spin; Komfort (comfort); & $16 \%$ each \\
9. Comfort, 10. Handling & Handling & $8 \%$ \\
Required Skill & Investitionsbedarf (required skill) & $5 \%$ \\
Sweetspot Size & Größe Sweetspot (sweetspot size) & $3 \%$ each \\
Crisp, Lively, Grip Size & crisp, Lebendigkeit (lively), Griffgröße (grip size) & \\
\hline
\end{tabular}


Table 3. Friedman's two-way ANOVA $p$-values indicating discrimination of certain attributes.

\begin{tabular}{cc}
\hline Sensory Attribute & $p$-Value \\
\hline Power & 0.236 \\
Control & 0.135 \\
Vibration & 0.067 \\
\hline
\end{tabular}

The fact that discrimination of vibration has been superior to power and control can also be observed when analyzing the mean range used by the testers for different attributes (Figure 1a). Using a smaller range within the available scale suggests that testers had more difficulty perceiving differences between rackets for that specific attribute. Another striking result is that the inter-rater agreement correlates with the mean range of the scale used by testers $(r=0.63, p=0.0504)$, even though the inter-rater agreement is only taking ranks into account, rather than the relative differences on the scale. The degree of agreement of several attributes, calculated by Fleiss' kappa, is shown in Figure $1 b$.

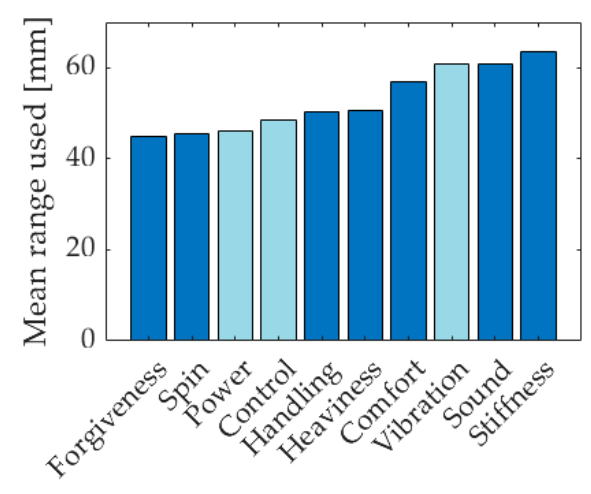

(a)

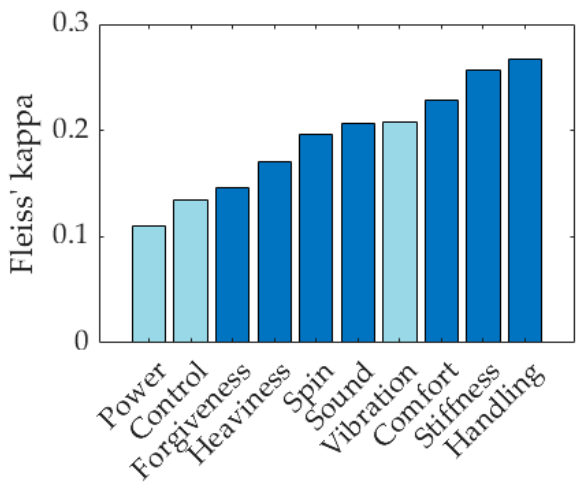

(b)

Figure 1. Bar plots for the 10 prominent sensory attributes of (a) mean range of the available scale used by testers and (b) Fleiss' kappa values (power, control, and vibration highlighted in light blue).

Industry-generated terms, such as power and control, lack inter-rater agreement and discrimination. This can be further illustrated by MFA correlation circles and corresponding profile plots of power (Figure 2a) and vibration (Figure 2b). In MFA correlation circles, close agreement is represented by vectors pointing in the same direction, whereas opposing directions indicate disagreement. Each vector represents one tester. Even though there seems to be an accumulation of power agreement at 3 o'clock in the MFA correlation circle, many outliers can be detected. This is further illustrated by random trends in the corresponding profile plots. There are fewer outliers for vibration; the vibration profile plot shows that agreement for rackets $A$ and $D$ is relatively high, with VAS ratings mostly ranging from 60 to 90 and 10 to $40 \mathrm{~mm}$, respectively.

The results of the PCA are presented in the form of a variables factor map in Figure 3, which illustrates the sensory profiles of the rackets used in this study. The first two principal components explain more than $90 \%$ of the total variance within the data and are therefore enough to visualize the correlation between the attributes. Black vectors refer to sensory attributes, whereas the blue vectors refer to additional variables, namely, the standardized rank sums of overall liking and racket specifications. The first dimension is best explained by control, handling, and overall (liking) as opposed to vibration (all $\cos ^{2}>0.85$ ). Sound appeal and balance best explain the second dimension. 

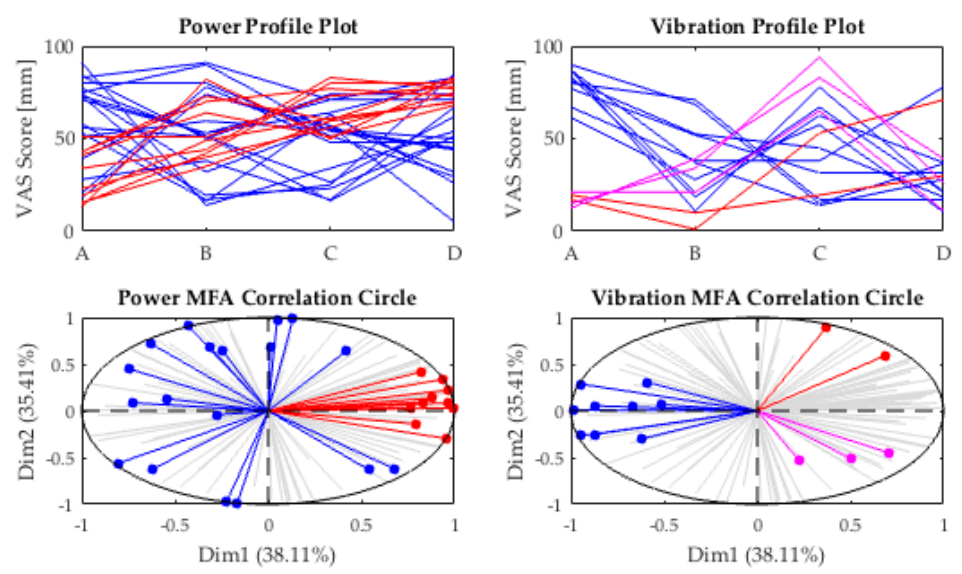

(a)

(b)

Figure 2. Profile plots, where each line represents the four ratings of one tester, and multiple factor analysis (MFA) correlation circles of (a) power and (b) vibration with particular clusters highlighted in blue, red, and magenta.

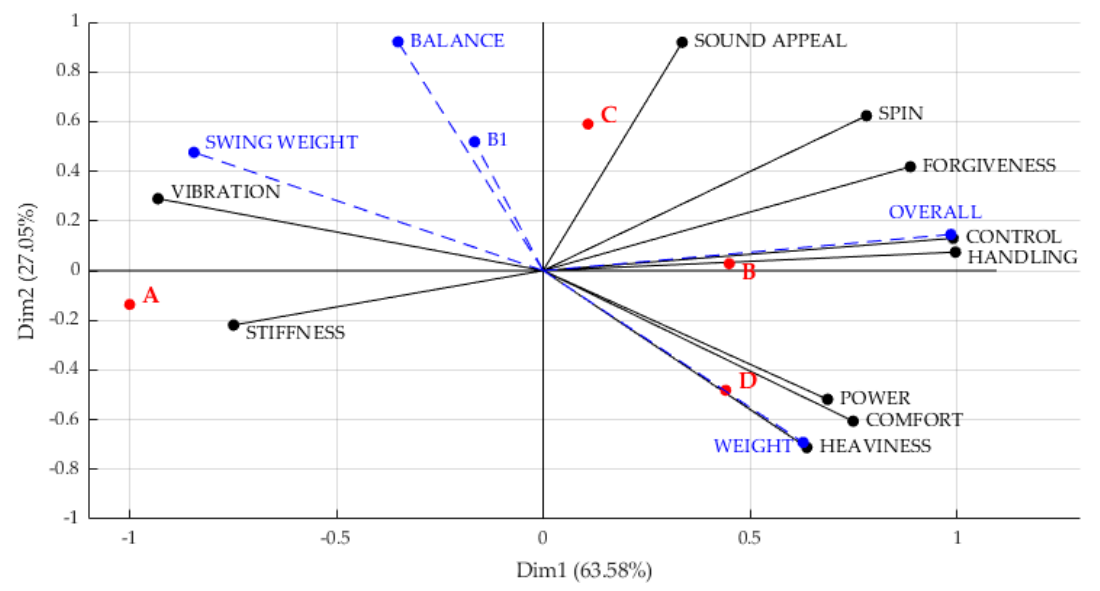

Figure 3. PCA variables factor map showing the relationship of the sensory attributes (black solid lines) with racket properties (blue dashed lines) and relative positioning of rackets A-D (red dots).

\section{Discussion}

The application of FCP has been successful in assessing the most prominent sensory attributes for this specific group of consumers as well as rapidly evaluating the sensory profile of this set of tennis rackets. It is not surprising that power and control were the most commonly used attributes, considering their presence in the marketing of tennis rackets. Attributes referring to physical characteristics, such as vibration or stiffness, have been used less often but also play a significant role in the perceived quality. Many descriptors refer to the playability of the racket, being described by forgiveness, handling, required skill, or size of the sweetspot. Impact- or feedback-specific ratings were mostly explained by vibration and comfort, rather than crisp or lively. Unsurprisingly, the previously identified feel parameters cosmetics and string bed [2] have not been used, as all rackets were coated black and strung at the same tension. It is, however, surprising that the relatively different dimensions or geometries of the rackets were not mentioned.

Even though PCA is not the common choice for statistical analysis of FCP data, it is effective in giving a comprehensive overview of relationships and sensory profiles of rackets. While racket $\mathrm{D}$ can be described as comfortable and heavy with easy access to power, racket B features good control and handling and racket $\mathrm{C}$ emits the most appealing sound. Racket $\mathrm{A}$ is the least liked, probably due to excessive levels of vibration and stiffness. For this specific group of players, control and handling seem to be the most important attributes when judging overall liking, whereas high levels of vibration 
and stiffness negatively affect the overall liking. The first bending mode does not show any relation to perceived stiffness or vibration, even though the differences are relatively large. Perceived heaviness seems to correlate with the mass of the rackets, rather than swing weight or balance. One possible explanation may be that differences between the rackets' swing weight are not perceivable.

One disadvantage of PCA is that its results are affected by the samples under investigation. There seems to be a positive relationship between racket mass and perceived power, which, however, could be artificial. The fact that the heaviest racket felt most powerful could be due to another factor. Moreover, PCA does not take inter-tester variance into account, as input scores are mean values. MFA, on the other hand, uses every single score and accounts for different frequencies in the use of attributes. Therefore, it is the more popular choice when analyzing FCP data. However, if agreement among testers is low, data visualization might get confusing. It is therefore suggested to use both PCA and MFA for analyzing subjective data in racket studies when using FCP.

Data analysis using PCA and MFA should always be accompanied by measures of reliability. Power and control appear as major contributors when characterizing the principal component space. However, these results should be interpreted with care, as inter-rater agreement and discrimination are both poor. The results suggest that tennis players are more sensitive to parameters referring to physical characteristics, such as vibration, rather than complex constructs, such as power or control, and can verbalize their perception on these parameters more consensually. Power is generally related to a racket's ability to generate high ball speeds, whereas control refers to the accuracy of shot placement. However, racket consumers seem to disagree on their exact definition. It is therefore suggested to investigate the general dimensions of power and control in more detail and to divide them into more clearly defined sub-themes, increasing agreement and reliability.

Even though this method allowed efficient profiling of the sensory characteristics of the rackets, the reliability and meaningfulness of subjective results in tennis racket testing require further research. The effect of different assessment types, such as pairwise comparison on reliability or the potential benefit of a trained panel, should be further investigated.

\section{Conclusions}

The application of FCP in sensory tennis racket analysis has proven successful in determining the most prominent attributes of the rackets under investigation, rapidly profiling them according to these attributes, establishing relationships between these attributes and identifying sources of the lack of agreement between testers in subjective evaluation of tennis rackets. Power, control, and vibration were the most commonly used attributes; however, tennis players seem to disagree on the exact meaning of power and control while judgment of attributes related to physical characteristics is more reliable. Even though FCP has been proven successful as a method for consumer research in the tennis racket industry, the use of trained panels, to potentially enhance reliability, and the effect of the assessment type on reliability must be explored.

Acknowledgments: The authors would like to thank HEAD Sport GmbH for the support of this study.

Conflicts of Interest: The funding sponsor assisted in the design of the study, mainly in the selection of the rackets under investigation, and in the data collection but had no role in the analyses or interpretation of data, in the writing of the manuscript, or in the decision to publish results.

\section{References}

1. Roberts, J.; Jones, R.; Harwood, C.; Mitchell, S.; Rothberg, S. Human perceptions of sports equipment under playing conditions. J. Sports Sci. 2001, 19, 485-497, doi:10.1080/026404101750238944.

2. Statham, A. Subjective and Objective Assessment of Tennis Racket Performance Play. Ph.D. Thesis, Loughborough University, Loughborough, UK, 2007.

3. Banwell, G. Impulsive Sound Quality of Tennis Rackets. Ph.D. Thesis, Loughborough University, Loughborough, UK, 2013.

4. Ares, G.; Varela, P. Trained vs. consumer panels for analytical testing: Fueling a long lasting debate in the field. Food Qual. Prefer. 2017, 61, 79-86, doi:10.1016/j.foodqual.2016.10.006. 
5. Varela, P.; Ares, G. Sensory profiling, the blurred line between sensory and consumer science. A review of novel methods for product characterization. Food Res. Int. 2012, 48, 893-908, doi:10.1016/j.foodres. 2012.06.037.

6. Williams, A.A.; Arnold, G.M. A comparison of the aromas of 6 coffees characterized by conventional profiling, free-choice profiling and similarity scaling methods. J. Sci. Food Agric. 1985, 36, 204-214, doi:10.1002/jsfa.2740360311.

7. Lê, S.; Josse, J.; Husson, F. FactoMineR: An R Package for Multivariate Analysis. J. Stat. Softw. 2008, 25, 1-18.

(C) 2020 by the authors. Licensee MDPI, Basel, Switzerland. This article is an open access article distributed under the terms and conditions of the Creative Commons Attribution (CC BY) license (http://creativecommons.org/licenses/by/4.0/). 